\title{
ST2L expression on monocyte subsets is associated with the severity of acute coronary syndrome and plaque vulnerability
}

\section{Type}

Research paper

\section{Keywords}

acute coronary syndrome, ST2, macrophage polarization, monocyte subset, plaque progression

\begin{abstract}
Introduction

Pro-inflammatory monocyte infiltration and conversion to macrophages is an established risk factor for acute coronary syndrome (ACS) and plaque instability visualized by optical coherence tomography (OCT). Increased serum levels of soluble ST2 isoform (SST2) predict poor outcomes in patients with ACS. However, whether the transmembrane ST2 isoform (ST2L) could early assess the severity of ACS and the vulnerability of plaques remains elusive.

\section{Material and methods}

Flow cytometry and ELISA were performed to evaluate the levels of ST2L on different monocyte subsets in ACS patients and serum concentration of SST2. Bone marrow-derived macrophages (BMDMs) were isolated and polarized to M1 or M2 phenotype, which levels of ST2L expression were detected by Quantitative RT-PCR and Immunoblots.

\section{Results}

The proportion and absolute number of ST2L on monocyte subsets 1 (Mon1) and Mon2 significantly declined, accompanied with increased serum SST2, in ACS patients comparing with controls. However, only proportion of ST2L+/Mon2 remained closely associated with the severity of atherosclerotic plaques. Patients with thin cap fibrous atheroma (TCFA) calculated by OCT had lower absolute number (Non-TCFA $1.26 \times 104 \pm 0.63 \times 104 / \mathrm{ml}$ vs. TCFA $0.92 \times 104 \pm 0.37 \times 104 / \mathrm{ml}, \mathrm{P}=0.034$ ) and lower percentage (Non-TCFA $17.65 \% \pm 3.10 \%$ vs. TCFA $13.17 \% \pm 2.29 \%, P<0.001$ ) of ST2L+/Mon2 than those without TCFA. In multivariate analysis, the proportion of ST2L+/Mon2 was considered as an independent determinant for TCFA. In BMDMs, SST2 and ST2L were highly expressed in M2 macrophage.
\end{abstract}

\section{Conclusions}

Compared to circulating SST2, the proportion of ST2L expressing on Mon2 subsets was more strongly associated with the severity of ACS and plaque vulnerability. 


\section{ST2L expression on monocyte subsets is associated with the severity of acute coronary syndrome and plaque vulnerability}

Peinan Ju, MD; Jianhui Zhuang, MD, PhD*; Shekhar Singh, MD; Yuyan Lyu, MD; Xiankai Li, MD, PhD*; Yawei Xu, MD, PhD

AFFILIATION: Department of Cardiology, Shanghai Tenth People's Hospital, Tongji University School of Medicine, Shanghai

*Corresponding authors: Jianhui Zhuang, MD, $\mathrm{PhD}, \mathrm{Email}$ : jh_zhuang@tongji.edu.cn; or to Xiankai Li, MD, PhD, Email: lixiankai@tongji.edu.cn; Department of Cardiology, Shanghai Tenth People's Hospital, Tongji University School of Medicine, 301 Middle Yanchang Road, Shanghai 200072, China

Runnning Title: ST2L and plaque vulnerability 


\begin{abstract}
Introduction: Pro-inflammatory monocyte infiltration and conversion to macrophages is an established risk factor for acute coronary syndrome (ACS) and plaque instability visualized by optical coherence tomography (OCT). Increased serum levels of soluble ST2 isoform (sST2) predict poor outcomes in patients with ACS and heart failure. However, whether the transmembrane ST2 isoform (ST2L) could early assess the severity of ACS and the vulnerability of plaques remains elusive.

Methods: Flow cytometry and ELISA were performed to evaluate the levels of ST2L on different monocyte subsets in ACS patients and serum concentration of sST2. Bone marrow-derived macrophages were isolated and then polarized to M1 or M2 phenotype, which levels of ST2L expression were detected by Quantitative RT-PCR and Immunoblots.
\end{abstract}

Results: We observed that the proportion and absolute number of ST2L on monocyte subsets 1 (Mon1) and Mon2 significantly declined, accompanied with increased serum sST2, in ACS patients as compared to controls. However, only proportion of $\mathrm{ST}_{2} \mathrm{~L}^{+} / \mathrm{Mon} 2$ remained closely associated with the severity of atherosclerotic plaques. With regard to the plaque instability, patients with thin cap fibrous atheroma (TCFA) calculated by optical coherence tomography (OCT) had lower absolute number (NonTCFA $1.26 \times 10^{4} \pm 0.63 \times 10^{4} / \mathrm{ml}$ vs. TCFA $\left.0.92 \times 10^{4} \pm 0.37 \times 10^{4} / \mathrm{ml}, \mathrm{P}=0.034\right)$ and lower percentage (Non-TCFA $17.65 \% \pm 3.10 \%$ vs. TCFA $13.17 \% \pm 2.29 \%, \mathrm{P}<0.001$ ) of $\mathrm{ST}_{2} \mathrm{~L}^{+} / \mathrm{Mon} 2$ than those without TCFA. In multivariate analysis, the proportion of $\mathrm{ST}_{2} \mathrm{~L}^{+} / \mathrm{Mon} 2$ was considered as an independent determinant for TCFA. In bone marrow-derived macrophages, sST2 and ST2L were highly expressed in M2 macrophage. 
Conclusions: Compared to circulating sST2, the proportion of ST2L expressing on Mon2 subsets was more strongly associated with the severity of ACS and plaque vulnerability.

Keywords: acute coronary syndrome, macrophage polarization, monocyte subset, plaque progression, ST2 


\section{Introduction}

Acute coronary syndrome (ACS) is referred to be one of the most important causes of mortality and morbidity worldwide.[1] It is widely accepted that plaque rupture or erosion followed by obstructive thrombus formation is the main features of ACS. Based on quantitative analysis of coronary angiography, it is evident that diverse parameters, including the presence of coronary plaque and the degree of luminal stenosis, carry incremental predictive value and improve risk stratification of ACS.[2] On the other hand, intravascular optical coherence tomography (OCT) is considered to generate high-resolution cross-sectional images of plaque morphology, allowing physicians to visualize the development of the distinct vulnerable plaques characterized by rupture-prone thickness of fibrotic cap and lipid rich necrotic core.[3] In addition, thin cap fibrous atheroma (TCFA) is more likely to have higher numbers of pro-inflammatory macrophages, with characteristics of punctate signalrich spots that exceed the background noise of the image by OCT.[4]

Suppression of tumorigenicity 2 (ST2), a member of interleukin 1 receptor (IL1R) family, constitutes two primary isoforms regulated by IL-33.[5, 6] The transmembrane ST2 receptor (ST2L) consists of a membrane-bound isoform with 3 extracellular IgG domain, while a soluble ST2 receptor (sST2), lacking transmembrane and intracellular domains, acts as an IL-33 decoy receptor and could be detected in circulating serum.[7] A growing evidence has expanded our knowledge about the biology function of IL-33/ST2 signaling and the pathogenesis of IL-33/ST2 in tumor development and cardiovascular abnormality.[8, 9] Depletion of ST2 in mice on pressure overload model results in excessive ventricular hypertrophy, chamber dilation and cardiac fibrosis.[10] Moreover, increased serum sST2 predicts poor 
outcomes in patients with ACS and chronic heart failure.[6, 11-14] By contrast, ST2L, predominantly expressed in macrophages, activates Th2 cells and mast cells, retards atherosclerotic development and alleviates cardiac remodeling through interaction of IL-33 and enhancement of its intracellular signaling.[15] However, the association of ST2L, especially ST2L on monocyte subsets, with the plaque characteristics remains unknown. Therefore, we aimed to determine the association of sST2 and ST2L on monocyte subsets with the severity and vulnerability of coronary plaques in the study.

\section{Materials and Methods}

The data that support the findings of this study are available from the corresponding author upon reasonable request.

\section{Study population}

Forty-eight patients with unstable angina pectoris (UAP) and 31 patients with STelevation myocardial infarction (STEMI) admitted to Shanghai Tenth People's Hospital from April 2013 and July 2014 were recruited as previously described.[16] UAP was diagnosed based on the manifestation of angina at rest, accelerated chest pain or new-onset chest pain without any abnormality of cardiac markers. STEMI was diagnosed according to the criteria of Fourth Universal Definition of Myocardial Infarction, [17] and further confirmed by coronary angiography with the presence of acute thrombosis secondary to plaque rupture. Thirty-three age- and sex-matched subjects with suspicious angina but free of luminal diameter narrowing $\geq 50 \%$ confirmed by angiography served as controls. Patients with severe liver and kidney failure, acute infectious disease and malignance were excluded. All patients provided written informed consent before enrollment. The study was approved by Shanghai 
Tenth People's Hospital and followed the guidelines of the Helsinki Declaration.

Quantification of plaque characteristics by angiography and optical coherence tomography

Coronary angiography and optical coherence tomography (OCT) were performed as previously described.[16] We defined the severity of ACS by evaluating the severity of coronary plaques obtained from coronary angiography. The severity of coronary plaques was evaluated by the length of plaque ( $>20 \mathrm{~mm}$ as long lesions), the degree of stenosis (moderate [from $50 \%$ to $69 \%$ ]; severe [from $70 \%$ to $99 \%$ ] or occlusion [100\%]) with or without calcification, and the number of coronary vessels with significant stenosis. Left main trunk stenosis was considered as a two-vessel disease. TCFA was defined as a plaque presenting lipid content for $>90$ degrees, and with thinnest part of the fibrous cap measuring $<65 \mu \mathrm{m}$ obtained from OCT.[18]

\section{Analysis of serum sST2}

About $5 \mathrm{ml}$ of peripheral venous blood was taken from controls and UAP patients at the day after fasting over 8 hours. Peripheral venous blood from patients with STEMI was collected and placed in EDTA-coated tubes at the day of charge. After centrifugation, serum was stored at $-80{ }^{\circ} \mathrm{C}$ for further analysis in batches. Serum levels of sST2 were measured with sandwich enzyme immunoassay using Human ST2/IL-33R Quantikine ELISA kits (catalog \#DST200, R\&D System, USA) according to the manufacture's protocol. The coefficients of variation $(\mathrm{CV})$ for intraassay precision and inter-assay precision were less than $5.2 \%$ and $6.8 \%$ respectively.

\section{Quantification of ST2L on monocyte subsets by flow cytometry}

For measurement of surface ST2L expression on monocytes, $100 \mu \mathrm{l}$ peripheral blood was blocked with $1 \%$ bovine serum albumin (BSA, Gibco, USA) solution for 15 
minutes at $4{ }^{\circ} \mathrm{C}$ and stained with a cocktail of monocyte antibodies for 30 minutes at 4 ${ }^{\circ} \mathrm{C}$. The information and concentration of each monocyte antibody is shown in Supplementary Table I. On basis of flow cytometry, circulating monocytes were divided into $\mathrm{CD} 14^{++} \mathrm{CD} 16^{-}$monocytes (Mon1), $\mathrm{CD} 14^{++} \mathrm{CD} 16^{+}$monocytes (Mon2) and $\mathrm{CD} 14^{+} \mathrm{CD} 16^{+}$monocytes (Mon3). PerCP-eFluor710-conjugated antibodies against IL-33R(ST2) were used to analyze the expression of ST2 receptors on monocytes (Figure 1). The flow cytometry for identifying surface ST2L expression and monocyte subsets was performed by one expert technician who was blind to the coronary angiography and OCT results.[16]

\section{Induction of macrophage polarization}

Animal studies were conducted in accordance with the Guide for the Care and Use of Laboratory Animals (U.S. National Institutes, MD, USA), and all the procedures were approved by the Animal Care and Use Committee of Shanghai Tenth People's Hospital. Bone marrow cells were isolated from femurs of C57BL/6 mice following standard protocol in Dulbecco's modified eagle's medium (DMEM, Gibco, USA) containing $10 \%$ fetal bovine serum (FBS, Gibco, USA) and 1\% penicillin and streptomycin.[19] Following separation, bone marrow-derived macrophages (BMDMs) were cultured in 10\% FBS/DMEM with recombinant M-CSF at a concentration of $100 \mathrm{ng} / \mathrm{ml}$ (Peprotech Incorporation, USA) for 3 days (M0 phenotype). BMDMs were washed and treated for 12 hours with DMEM $+5 \%$ FBS alone, LPS at a concentration of $100 \mathrm{ng} / \mathrm{ml}$ and recombinant interferon gamma (IFN$\gamma$, Peprotech Incorporation, USA) at a concentration of $20 \mathrm{ng} / \mathrm{ml}$ for polarization to the M1 phenotype, or recombinant IL-4 at a concentration of $20 \mathrm{ng} / \mathrm{ml}$ (Peprotech Incorporation, USA) for polarization to the M2 phenotype. Likewise, human 
circulating monocytes were collected from healthy volunteers, differentiated into macrophages and maintained RPMI 1640 medium (Gibco, USA) supplemented with $10 \%$ FBS according to previous protocols.[20]

\section{Quantitative RT-PCR}

Total cellular RNA was extracted and purified using Trizol reagent (Invitrogen, USA). Reverse transcription was performed using Transcriptor First Strand cDNA Synthesis Kit (Roche, Germany). Quantitative RT-PCR for ST2L, TNF- $\alpha$, IL-6, CD206 and Arg1 was performed using FastStart Universal SYBR Green Master (Roche, Germany) on a Roche Lightcycler. The primer sequences predesigned for quantitative RT-PCR are tabulated in Supplementary Table II.

\section{Immunoblots}

Protein lysates were performed on $8 \%$ SDS-PAGE gels and transferred to membranes as our previously reported.[21] The membranes were probed using antibody directed against ST2L (Abcam, USA) and normalized to GAPDH (Santa Cruz, USA).

\section{Statistical analysis}

Continuous variables were presented as mean \pm standard deviation (SD). Categorical variables were presented as absolute number and proportion. Differences between two groups were analyzed with Student's t test. Differences between multiple groups were initially compared using One-way ANOVA test and post-hoc tests were then performed with Bonferroni comparison. Receiver-operating characteristics (ROC) curves were conducted to determine the performance of serum sST2 and ST2L expression on monocytes in plaque vulnerability. Youden's index was applied to derive the best cut-off value of ST2L expression on monocytes. Based on the cut-off, we then performed multivariate logistic regression backward stepwise that included 
variables with $\mathrm{P}<0.1$ from univariate test to identify independent predictors of the plaque vulnerability. Values of $P<0.05$ were considered significant.

\section{Results}

\section{Comparison of serum SST2 and ST2L on monocytes among different severity of}

\section{ACS}

The patient characteristics, angiographic findings and results of OCT imaging were summarized in our previous study.[16] Of the whole measurements, the proportions and the absolute number of ST2L on Monocytes and Mon2 subsets in STEMI patients were significantly lower than those in UAP patients and controls (Table I). Consistent with other researches, serum levels of sST2 showed a steady upward from controls to STEMI patients (Control $1.35 \pm 0.65 \mathrm{ng} / \mathrm{ml}$, UAP $2.42 \pm 0.61 \mathrm{ng} / \mathrm{ml}$ and STEMI 3.88 $\pm 1.61 \mathrm{ng} / \mathrm{ml}, P<0.001)$.

Based on quantitative analysis of coronary angiography, it is evident that diverse parameters, including the presence of coronary plaque and the degree of luminal stenosis, carry incremental predictive value and improve risk stratification of ACS.[2] We next measured the association of serum sST2 and ST2L on monocyte subsets with the severity of ACS detected by angiography. As a result, there was no markedly association between serum sST2 and the atherosclerotic severity of ACS (Figure 2A, $2 \mathrm{E}$ and $2 \mathrm{I}$ ). ACS patients with lower proportion of $\mathrm{ST}_{2} \mathrm{~L}^{+} / \mathrm{Mon}$ and $\mathrm{ST} 2 \mathrm{~L}^{+} / \mathrm{Mon} 1$ were more likely than those with higher proportion to have obstructive stenosis (Figure 2B, $2 \mathrm{~F}$ and $2 \mathrm{~J}$ ). It should be noted that the percentage of ST2L ${ }^{+} / \mathrm{Mon} 2$ significantly inversely correlated with all of three angiographic parameters (Figure 2D, $2 \mathrm{H}$ and $2 \mathrm{~L}$ ). 


\section{stability}

Plaque characteristics by OCT were summarized in our previous study. Of the OCT subgroup, patients with TCFA had lower absolute number (Non-TCFA $1.26 \times 10^{4} \pm$ $0.63 \times 10^{4} / \mathrm{ml}$ vs. TCFA $\left.0.92 \times 10^{4} \pm 0.37 \times 10^{4} / \mathrm{ml}, \mathrm{P}=0.034\right)$ and lower percentage (Non-TCFA $17.65 \% \pm 3.10 \%$ vs. TCFA $13.17 \% \pm 2.29 \%, \mathrm{P}<0.001)$ of $\mathrm{ST}_{2} \mathrm{~L}^{+} / \mathrm{Mon} 2$ than those without TCFA (Table II). However, there was no difference in serum levels of sST2 between groups (Table II). We then analyzed the association of serum sST2 and ST2L on monocytes with plaque morphology, and found that serum SST2 was positively correlated with the thickness of fibrotic cap (Pearson $r=0.306, P=0.034$ ), while no discrepancies of serum sST2 in arc of lipid core and minimum lumen area were observed (Table III). On the contrary, percentage of ST2L $\mathrm{L}^{+} / \mathrm{Mon} 2$ was gradually increased as the fibrous cap thickness grew (Pearson $\mathrm{r}=0.523, \mathrm{P}<0.001$ ), whilst there was an inverse correlation between percentage of ST2 $\mathrm{L}^{+} / \mathrm{Mon} 2$ and $\operatorname{arc}$ of lipid core $($ Pearson $\mathrm{r}=-0.473, \mathrm{P}=0.001)$

\section{ST2L on Mon2 as determinants of plaque vulnerability}

The under area of ROC curve of percentage and absolute number of ST2 $\mathrm{L}^{+} / \mathrm{Mon} 2$ for determining TCFA was 0.889 and 0.663 , respectively (Figure 3). The optical percentage of ST2 $\mathrm{L}^{+} / \mathrm{Mon} 2$ cutoff to predict TCFA was $15.31 \%$, which had sensitivity of 82.41 , specificity of 85.71 (Figure 3 ).

After correction for known clinical risk factors including age, gender, smoking, hypertension, diabetes mellitus, white blood cells (WBC), Low-Density Lipoprotein Cholesterol (LDL-C), and the percentage of circulating Mon2 subsets, decreased levels of percentage of ST2L $\mathrm{L}^{+} / \mathrm{Mon} 2$ remained an independent predictor of TCFA (OR 0.07, 95\% CI 0.02-0.29, $\mathrm{P}<0.001$, Table IV). 


\section{ST2L was primary expressed in M2 macrophages}

It is well known that with the development of atherosclerosis, BM-derived circulating monocytes possess the capacity to differentiate into two macrophage subsets based on their pathologic function and expressions of divergent cytokines.[22] To examine the abundance of ST2L on the macrophage subsets, we induced BMDMs and human macrophages by different stimuli, and confirmed the results by quantitative PCR and immunoblots (Figure 4A). As compared to M1 macrophages, both mRNA and protein of ST2L was more abundant in M2 polarized macrophages, suggesting the likelihood of ST2L in regulating plaque instability (Figure 4B-D). Interestingly, we found that the concentration of sST2 in the supernatant of M2 macrophages was higher than that of M1 macrophages differentiated from human circulating monocytes (Figure 4E).

\section{Discussion}

To our best knowledge, we indicate for the first time that ST2L expressing on Mon2, particularly the percentage of ST2L $\mathrm{L}^{+} / \mathrm{Mon} 2$ in circulating leukocytes, was tightly associated with the incidence and severity of ACS. The percentage of $\mathrm{ST}_{2} \mathrm{~L}^{+} / \mathrm{Mon} 2$, rather than serum sST2, seemed more likely to be a preferred biomarker reflecting the signature of plaque destabilization detected by OCT.

It has been reported that IL-33 was predominantly produced by vascular smooth muscle cells and endothelial cells in response to atherogenic stimuli.[8] Likewise, cardiac hypertrophy and mechanical strain substantially induced IL-33 expression, most of which was abundant in cardiac fibroblasts.[10] Mechanistically, through neutralization against IL-33, sST2 abrogated the cardioprotective effect of IL-33/ST2 signaling in cardiac fibrosis and hypertrophy.[11] While sST2 acted as a circulating 
isoform and was ubiquitously expressed in various tissues, the transmembrane isoform ST2L was inducible and primarily located in hematopoietic cells, especially Th2 cells, mast cells, monocytes and macrophages.[23] In the present study, we found that ST2L was highly expressed in M2 macrophage, suggesting that ST2L might be involved in M2 macrophage activation, which contributes to angiogenesis and antiinflammation. Consistently, a previous research observed that depletion of ST2L disrupts macrophage polarization to M2 phenotype with decreased M2 macrophage markers.[15] In parallel, the supernatant of M2 macrophages had a higher concentration of sST2 than that of M0 and M1 macrophages.

Weinberg et al.[6], for the first time, revealed that elevated serum sST2 predicted higher likelihood of 30-day mortality and heart failure in patients with myocardial infarction. Likewise, while no-reflow phenomenon reflects impaired myocardial perfusion and poor prognosis[24], including mortality and heart failure, in ACS patients after percutaneous coronary intervention, Somuncu et al.[25] showed serum sST2 as an independent predictor of no-reflow phenomenon in ACS patients. A recent meta-analysis summarized that ACS patients with higher baseline levels of serum sST2 predicted the increased possibility of all-cause mortality and major adverse cardiac events during follow-up.[13] In parallel, our study confirmed that serum sST2 level was higher in ACS patients as compared with controls. Nevertheless, there was no statistical discrepancy in serum SST2 between UAP patients with and without TCFA, implying that serum sST2 might not be considered as a predictor of plaque destabilization.

Nonetheless, little is understood about the association of ST2L with the incidence of ACS and plaque vulnerability. Circulating monocytes extravasate through the 
endothelium and differentiate into two opposite macrophage subsets, initiating atherosclerosis and regulating plaque stabilization.[26] To be more specific, $\mathrm{CD} 14^{++} \mathrm{CD} 16^{-}$Mon1 subsets and $\mathrm{CD} 14^{++} \mathrm{CD} 16^{+}$Mon2 subsets more readily invade subendothelium and differentiate to active M1 macrophages, which subsequently contribute to necrotic core formation, matrix degradation and plaque rupture. Others' and our previous studies have shown that $\mathrm{CD} 14^{++} \mathrm{CD} 16^{+}$Mon2 subsets were tightly associated with the incidence of ACS.[16, 27] Furthermore, higher CD14 ${ }^{++} \mathrm{CD} 16^{+}$ Mon2 subsets were more often found in patients with TCFA.[16] Therefore, together with the involvement of ST2L in macrophage polarization, these data supported us further investigation whether ST2L on monocyte subsets could be considered as a potential biomarker for ACS and plaque instability. In this regard, we firstly observed that ST2L was highly expressed in Mon1 and Mon2 subsets, relatively lower in Mon3 subsets, suggesting that ST2L mainly functioned in pro-inflammatory monocytes which subsequently polarized to M1 macrophages and contributed to plaque development and rupture. Our study next provides evidence for higher percentage of $\mathrm{ST}_{2} \mathrm{~L}^{+} / \mathrm{Mon} 2$ as a determinant for ACS. On the other hand, numerous clinical trials propose that integrated measurements of plaque composition, location and extent based on coronary computed tomography angiography or invasive coronary angiography is capable of accurate risk stratification and prognosis for ACS patients. $[2,28]$ In this regard, only proportion of $\mathrm{ST}_{2} \mathrm{~L}^{+} / \mathrm{Mon} 2$ was prevalently lower in patients with high-risk plaque features, indicating that proportion of ST2L $\mathrm{L}^{+} / \mathrm{Mon} 2$ might be referred as a promising biomarker for risk stratification of ACS.

Given the high image resolution with OCT, it allows physicians to identify highrisk plaques in vivo. We found that, in ACS patients, higher percentage of 
ST2L ${ }^{+} /$Mon2 indicated thinner fibrotic cap accompanied with larger extent of lipid core, both of which serve as early features of plaque instability.[29] Combined with ROC curve and multivariate analysis, we further accentuated the superiority of the percentage of ST2L $\mathrm{L}^{+} / \mathrm{Mon} 2$ against other clinical factors in predicting vulnerable plaques. From the results of our previous study, circulating Mon2 subset was considered as an independent predictor of the incidence of definite TCFA. It is therefore plausible to assume that ST2L especially expressing on Mon2 subset, depending on its diverse pathogenic roles and clinical significances in atherosclerosis and myocardial infarction, further collaborated to optimize the prediction of plaque vulnerability.

Finally, we must confess some potential limitations in our research. In the current study, we did not investigate the distribution of ST2L on Th2 in ACS patients. It has been proven that accumulation of pro-inflammatory macrophages in infarct myocardium and phagocytosis of necrotic cardiomyocytes by macrophages refer to a hallmark during early phase of myocardial infarction.[30] While Th1 subsets seems more likely to affect the evolution of ACS, the absolute number of circulating Th2 subsets is relatively low and there is no prominent difference in circulating Th2 subsets between patients with stable angina and ACS.[31] Despite all this, a comprehensive assessment of ST2L on Th2 may boost the understanding of IL33/ST2 signaling in atherosclerotic development. Particularly, a larger number of patients in another external validation cohort was required to strengthen our findings. In multivariate logistic regression analysis for predictors of TCFA, several wellknown risk factors including diabetes, WBC, LDL-C didn't show significant effects on TCFA as what previous studies have shown[32-34], which may due to the small 
size of our study. Moreover, accumulating evidence in the functional characterization of macrophages has revealed that the phenotypes are not limited to the M1 and M2 extremes but rather represent a continuous spectrum of phenotypes associated with differential cytokine production and functional characteristics.[35] Given the difficulty in simulating the environment of macrophages in plaques in vitro, we refer to the concepts of M1 and M2 in this study, taking M1 to primarily represent macrophages showing proinflammatory phenotype and M2 to represent antiinflammatory phenotype.

\section{Conclusions}

Enhanced proportion of ST2 $\mathrm{L}^{+} / \mathrm{Mon} 2$ was related to the severity of ACS and plaque vulnerability in coronary artery. ST2 $\mathrm{L}^{+} / \mathrm{Mon} 2$ might constitute a promising biomarker for the early diagnosis of vulnerable plaques.

\section{Acknowledgment}

We appreciate patients who participated in this study. This study is supported by Grant No. 81800424 and No. 81800378 from National Natural Science Foundation of China, and by the Fundamental Research Funds for the Central Universities (No. 22120180581). 


\section{References}

1. Ibanez B, James S, Agewall S, et al. 2017 ESC Guidelines for the management of acute myocardial infarction in patients presenting with ST-segment elevation: The Task Force for the management of acute myocardial infarction in patients presenting with ST-segment elevation of the European Society of Cardiology (ESC). Eur Heart J. 2018; 39:119-177.

2. Hadamitzky M, Achenbach S, Al-Mallah M, et al. Optimized prognostic score for coronary computed tomographic angiography: results from the CONFIRM registry (COronary CT Angiography Evaluation For Clinical Outcomes: An InteRnational Multicenter Registry). J Am Coll Cardiol. 2013;62:468-76.

3. Sinclair H, Bourantas C, Bagnall A, Mintz GS and Kunadian V. OCT for the identification of vulnerable plaque in acute coronary syndrome. JACC Cardiovasc Imaging. 2015; 8:198-209.

4. Cassar A, Matsuo Y, Herrmann J, et al. Coronary atherosclerosis with vulnerable plaque and complicated lesions in transplant recipients: new insight into cardiac allograft vasculopathy by optical coherence tomography. Eur Heart J. 2013;34:26107.

5. Schmitz J, Owyang A, 01dham E, et al. IL-33, an interleukin-1-1ike cytokine that signals via the $\mathrm{IL}^{-1}$ receptor-related protein $\mathrm{ST} 2$ and induces $\mathrm{T}$ helper type $2^{-}$ associated cytokines. Immunity. 2005;23:479-90.

6. Weinberg E0, Shimpo M, De Keulenaer GW, et al. Expression and regulation of ST2, an interleukin-1 receptor family member, in cardiomyocytes and myocardial infarction. Circulation. 2002;106:2961-6.

7. Liew FY, Pitman NI and McInnes IB. Disease-associated functions of IL-33: the new kid in the IL-1 family. Nat Rev Immunol. 2010;10:103-10.

8. Pascual-Figal DA and Januzzi JL. The biology of ST2: the International ST2 Consensus Pane1. Am J Cardiol. 2015;115:3B-7B.

9. Andersson P, Yang Y, Hosaka K, et al. Molecular mechanisms of IL-33-mediated stromal interactions in cancer metastasis. JCI Insight. 2018;3.

10. Veeraveedu PT, Sanada S, Okuda K, et al. Ablation of IL-33 gene exacerbate myocardial remodeling in mice with heart failure induced by mechanical stress. Biochem Pharmacol. 2017;138:73-80.

11. Sanada S, Hakuno D, Higgins LJ, Schreiter ER, McKenzie AN and Lee RT. IL-33 and ST2 comprise a critical biomechanically induced and cardioprotective signaling system. J Clin Invest. 2007;117:1538-49.

12. Ghali R, Altara R, Louch WE, et al. IL-33 (Interleukin 33)/sST2 Axis in Hypertension and Heart Failure. Hypertension. 2018;72:818-828.

13. Gu L and Li J. Short-term and long-term prognostic value of circulating soluble suppression of tumorigenicity-2 concentration in acute coronary syndrome: a metaanalysis. Biosci Rep. 2019;39.

14. Michalska-Kasiczak M, Bielecka-Dabrowa A, von Haehling S, Anker SD, Rysz J and Banach M. Biomarkers, myocardial fibrosis and co-morbidities in heart failure with preserved ejection fraction: an overview. Arch Med Sci. 2018;14:890-909.

15. Miller AM, Xu D, Asquith DL, et al. IL-33 reduces the development of atherosclerosis. J Exp Med. 2008;205:339-46.

16. Zhuang J, Han $\mathrm{Y}$, Xu D, et al. Comparison of circulating dendritic cell and monocyte subsets at different stages of atherosclerosis: insights from optical coherence tomography. BMC Cardiovasc Disord. 2017;17:270.

17. Thygesen K, Alpert JS, Jaffe AS, et al. Fourth Universal Definition of Myocardial Infarction (2018). J Am Coll Cardiol. 2018;72:2231-2264. 
18. Tearney GJ, Regar E, Akasaka T, et al. Consensus standards for acquisition, measurement, and reporting of intravascular optical coherence tomography studies: a report from the International Working Group for Intravascular 0ptical Coherence Tomography Standardization and Validation. J Am Coll Cardiol. 2012;59:1058-72.

19. Oteng AB, Ruppert PMM, Boutens L, et al. Characterization of ANGPTL4 function in macrophages and adipocytes using Angpt14-knockout and Angpt14-hypomorphic mice. J Lipid Res. 2019.

20. Finn AV, Nakano M, Polavarapu R, et al. Hemoglobin directs macrophage differentiation and prevents foam cell formation in human atherosclerotic plaques. J Am Coll Cardiol. 2012;59:166-77.

21. Luan P, Jian W, Xu X, et al. NLRC5 inhibits neointima formation following vascular injury and directly interacts with PPARgamma. Nat Commun. 2019;10:2882.

22. Murray PJ, Allen JE, Biswas SK, et al. Macrophage activation and polarization: nomenclature and experimental guidelines. Immunity. 2014;41:14-20.

23. Turnquist HR, Zhao Z, Rosborough BR, et al. IL-33 expands suppressive CD11b+ Gr1 (int) and regulatory $\mathrm{T}$ cells, including ST2L+Foxp3+ cells, and mediates regulatory $\mathrm{T}$ cell-dependent promotion of cardiac allograft survival. J Immunol. 2011;187:4598610 .

24. Valgimigli M, Campo G, Malagutti P, et al. Persistent coronary no flow after wire insertion is an early and readily available mortality risk factor despite successful mechanical intervention in acute myocardial infarction: a pooled analysis from the STRATEGY (Single High-Dose Bolus Tirofiban and Sirolimus-Eluting Stent Versus Abciximab and Bare-Metal Stent in Acute Myocardial Infarction) and MULTISTRATEGY (Multicenter Evaluation of Single High-Dose Bolus Tirofiban Versus Abciximab With Sirolimus-Eluting Stent or Bare-Metal Stent in Acute Myocardial Infarction Study) trials. JACC Cardiovasc Interv. 2011;4:51-62.

25. Somuncu MU, Akgun T, Cakir M0, et al. The Elevated Soluble ST2 Predicts NoReflow Phenomenon in ST-Elevation Myocardial Infarction Undergoing Primary Percutaneous Coronary Intervention. J Atheroscler Thromb. 2019.

26. Tabas I and Bornfeldt KE. Macrophage Phenotype and Function in Different Stages of Atherosclerosis. Circ Res. 2016;118:653-67.

27. Rogacev KS, Cremers B, Zawada AM, et al. CD14++CD16+ monocytes independently predict cardiovascular events: a cohort study of 951 patients referred for elective coronary angiography. J Am Coll Cardiol. 2012;60:1512-20.

28. Mancini GBJ, Hartigan PM, Shaw LJ, et al. Predicting outcome in the COURAGE trial (Clinical Outcomes Utilizing Revascularization and Aggressive Drug Evaluation): coronary anatomy versus ischemia. JACC Cardiovasc Interv. 2014;7:195-201.

29. Ahmadi A, Leipsic J, Blankstein $\mathrm{R}$, et al. Do plaques rapidly progress prior to myocardial infarction? The interplay between plaque vulnerability and progression. Circ Res. 2015; 117:99-104.

30. Yan X, Anzai A, Katsumata Y, et al. Temporal dynamics of cardiac immune cel1 accumulation following acute myocardial infarction. J Mol Cell Cardiol. 2013;62:2435.

31. Methe H, Brunner S, Wiegand D, Nabauer M, Koglin J and Edelman ER. Enhanced Thelper-1 lymphocyte activation patterns in acute coronary syndromes. J Am Coll Cardiol. 2005; 45:1939-45.

32. Hong YJ, Jeong MH, Choi YH, et al. Plaque characteristics in culprit lesions and inflammatory status in diabetic acute coronary syndrome patients. JACC Cardiovasc Imaging. 2009;2:339-49.

33. Raffel OC, Tearney GJ, Gauthier DD, Halpern EF, Bouma BE and Jang IK. Relationship between a systemic inflammatory marker, plaque inflammation, and plaque 
characteristics determined by intravascular optical coherence tomography. Arterioscler Thromb Vasc Biol. 2007;27:1820-7.

34. Nasu K, Terashima M, Habara M, et al. Impact of cholesterol metabolism on coronary plaque vulnerability of target vessels: a combined analysis of virtual histology intravascular ultrasound and optical coherence tomography. JACC Cardiovasc Interv. 2013;6:746-55.

35. Stoger JL, Gijbels MJ, van der Velden S, et al. Distribution of macrophage polarization markers in human atherosclerosis. Atherosclerosis. 2012;225:461-8. 


\section{Figure legends}

Figure 1. Gating strategy for circulating monocyte subsets and ST2L content Whole peripheral blood cells were prepared and labeled with various antibodies. CD14 was used in conjunction with CD16 to identify monocyte subsets (Mon). Mon1 was identified as $\mathrm{CD} 14^{++} \mathrm{CD} 16^{-}$, Mon2 as $\mathrm{CD} 14^{++} \mathrm{CD} 16^{+}$and Mon3 as $\mathrm{CD} 14^{+} \mathrm{CD} 16^{+}$. ST2L antibodies were further used to stratify the percentage of ST2L positive monocyte subsets.

Figure 2. Comparison of serum sST2 and the percentage of ST2L expression on monocytes in patients with different severities of coronary plaques detected by angiography

A-D. Comparison of serum SST2 and the percentage of ST2L expression on monocytes according to the number of stenotic vessels. E-H. Comparison of serum ST2 and the percentage of ST2L expression on monocytes according to the degree of stenosis. I-L. Comparison of serum ST2 and the percentage of ST2L expression on monocytes according to the lesion length. Abbreviations: ST2 = suppression of tumorigenicity $2, \mathrm{ST} 2 \mathrm{~L}=$ transmembrane ST2 isoform, Mon $=$ monocyte. ${ }^{*} P<0.05$.

Figure 3. ROC curve depicting the distinguishing ability of absolute number and percentage of ST2 $\mathrm{L}^{+} / \mathrm{Mon} 2$ for TCFA.

Abbreviations: $\mathrm{ROC}=$ receiver-operating characteristics; $\mathrm{AUC}=$ area under the curve; TCFA $=$ thin cap fibrous atheroma.

\section{Figure 4. ST2L accumulates in M2 macrophages}

A. Quantification of M1 and M2 markers in BM-derived macrophages. Macrophages were induced by LPS $(100 \mathrm{ng} / \mathrm{ml})$ and IFN- $\gamma(20 \mathrm{ng} / \mathrm{ml})$ for M1 polarization or by IL$4(20 \mathrm{ng} / \mathrm{ml})$ for M2 polarization. B and C. Quantitative PCR indicated that ST2L was upregulated in M2 polarized phenotype of mouse bone marrow-derived macrophages (B) and human macrophages (C). D. Immunoblots showed the protein expression of ST2L in M0, M1 and M2 macrophages differentiated from human circulating monocytes. E. Comparison of the sST2 levels between the supernatant of M1 and M2 macrophages differentiated from human circulating monocytes. $* P<0.05$. 
Table I. Comparison of serum sST2 and ST2L expression on monocytes among three groups

\begin{tabular}{lcccccc}
\hline & Control $(\mathrm{n}=33)$ & UAP $(\mathrm{n}=48)$ & STEMI $(\mathrm{n}=31)$ & P value & P (group1\&2) & P (group2\&3) \\
\hline sST2 $(\mathrm{ng} / \mathrm{ml})$ & $1.35 \pm 0.65$ & $2.42 \pm 0.61$ & $3.88 \pm 1.61$ & $<\mathbf{0 . 0 0 1}$ & $<\mathbf{0 . 0 0 1}$ & $<\mathbf{0 . 0 0 1}$ \\
ST2L ${ }^{+}$Mon $(\%)$ & $21.7 \pm 3.76$ & $14.9 \pm 1.83$ & $9.34 \pm 2.48$ & $<\mathbf{0 . 0 0 1}$ & $<\mathbf{0 . 0 0 1}$ & $<\mathbf{0 . 0 0 1}$ \\
ST2L ${ }^{+}$Mon1 $(\%)$ & $25.0 \pm 3.93$ & $16.8 \pm 2.39$ & $9.84 \pm 3.02$ & $<\mathbf{0 . 0 0 1}$ & $<\mathbf{0 . 0 0 1}$ & $<\mathbf{0 . 0 0 1}$ \\
ST2L $L^{+}$Mon2 $(\%)$ & $20.9 \pm 2.29$ & $15.8 \pm 3.56$ & $10.13 \pm 1.52$ & $<\mathbf{0 . 0 0 1}$ & $<\mathbf{0 . 0 0 1}$ & $<\mathbf{0 . 0 0 1}$ \\
ST2L $^{+}$Mon3 $(\%)$ & $5.13 \pm 1.30$ & $4.36 \pm 1.38$ & $4.89 \pm 1.24$ & $\mathbf{0 . 0 2 9}$ & $\mathbf{0 . 0 3 3}$ & 0.249 \\
ST2L $^{+}$Mon, $10^{4} / \mathrm{ml}$ & $9.17 \pm 4.28$ & $6.62 \pm 2.52$ & $6.41 \pm 2.64$ & $\mathbf{0 . 0 0 1}$ & $\mathbf{0 . 0 0 2}$ & $\mathbf{1 . 0 0 0}$ \\
ST2L $^{+}$Mon1, $10^{4} / \mathrm{ml}$ & $7.58 \pm 3.78$ & $5.23 \pm 2.08$ & $4.94 \pm 2.21$ & $<\mathbf{0 . 0 0 1}$ & $\mathbf{0 . 0 0 1}$ & $\mathbf{0 . 0 0 3}$ \\
ST2L $L^{+}$Mon2, $10^{4} / \mathrm{ml}$ & $1.30 \pm 0.63$ & $1.12 \pm 0.56$ & $1.16 \pm 0.49$ & 0.370 & & \\
ST2L $L^{+}$Mon3, $10^{3} / \mathrm{ml}$ & $2.88 \pm 1.87$ & $2.68 \pm 1.85$ & $3.11 \pm 1.61$ & 0.584 & & \\
\hline
\end{tabular}

Data are presented as mean \pm SD. P (group1\&2) indicates the difference between Control and UAP groups after post-hoc Bonferroni comparison. $\mathrm{P}$ (group2\&3) indicates the difference between UAP and STEMI groups after post-hoc Bonferroni comparison.

Abbreviations: UAP $=$ unstable angina pectoris, STEMI $=$ ST-elevation myocardial infarction, ST2 $=$ suppression of tumorigenicity 2, ST2L $=$ transmembrane ST2 isoform, Mon = monocyte. 
Table II. Comparison of serum SST2 and ST2L expression on monocytes in patients with and without TCFA

\begin{tabular}{lccc}
\hline & Non-TCFA $(\mathrm{n}=28)$ & TCFA $(\mathrm{n}=20)$ & P value \\
\hline sST2 $(\mathrm{ng} / \mathrm{ml})$ & $2.54 \pm 0.53$ & $2.24 \pm 0.69$ & 0.096 \\
ST2L $L^{+}$Mon $(\%)$ & $14.74 \pm 1.90$ & $15.03 \pm 1.77$ & 0.595 \\
ST2L $L^{+}$Mon1 (\%) & $16.29 \pm 2.45$ & $17.41 \pm 2.21$ & 0.110 \\
ST2L $L^{+}$Mon2 $(\%)$ & $17.65 \pm 3.10$ & $13.17 \pm 2.29$ & $<\mathbf{0 . 0 0 1}$ \\
ST2L $L^{+}$Mon3 $(\%)$ & $4.51 \pm 1.52$ & $4.14 \pm 1.14$ & 0.361 \\
ST2L $L^{+}$Mon, $10^{4} / \mathrm{ml}$ & $7.18 \pm 2.77$ & $5.84 \pm 1.93$ & 0.069 \\
ST2L $L^{+}$Mon1, $10^{4} / \mathrm{ml}$ & $5.59 \pm 2.29$ & $4.72 \pm 1.68$ & 0.153 \\
ST2L $L^{+}$Mon2, $10^{4} / \mathrm{ml}$ & $1.26 \pm 0.63$ & $0.92 \pm 0.37$ & $\mathbf{0 . 0 3 9}$ \\
ST2L $L^{+}$Mon3, $10^{3} / \mathrm{ml}$ & $3.20 \pm 2.08$ & $1.96 \pm 1.18$ & $\mathbf{0 . 0 2 1}$ \\
\hline
\end{tabular}

Data are presented as mean $\pm \mathrm{SD}$.

Abbreviations: TCFA $=$ thin cap fibrous atheroma. Other abbreviations listed as Table I. 
Table III. Correlation of serum sST2 and ST2L expression on monocytes with plaque morphology

\begin{tabular}{|c|c|c|c|c|c|c|}
\hline & \multicolumn{2}{|c|}{ Fibrous cap thickness } & \multicolumn{2}{|c|}{ Arc of lipid core } & \multicolumn{2}{|c|}{ MLA } \\
\hline & $\mathrm{r}$ & $\mathrm{p}$ value & $\mathrm{r}$ & $\mathrm{p}$ value & $\mathrm{r}$ & $\mathrm{p}$ value \\
\hline sST2 (ng/ml) & 0.306 & 0.034 & 0.076 & 0.608 & 0.257 & 0.078 \\
\hline ST2L ${ }^{+} \operatorname{Mon}(\%)$ & -0.239 & 0.101 & -0.099 & 0.505 & 0.078 & 0.599 \\
\hline ST2L $L^{+}$Mon $1(\%)$ & -0.245 & 0.094 & -0.245 & 0.094 & -0.123 & 0.405 \\
\hline ST2L $L^{+}$Mon $2(\%)$ & 0.523 & $<0.001$ & -0.473 & 0.001 & 0.265 & 0.069 \\
\hline ST2 $L^{+}$Mon3 $(\%)$ & 0.203 & 0.167 & 0.292 & 0.044 & 0.146 & 0.321 \\
\hline $\mathrm{ST} 2 \mathrm{~L}^{+}$Mon, $10^{4} / \mathrm{ml}$ & -0.010 & 0.947 & 0.165 & 0.262 & 0.054 & 0.714 \\
\hline ST2L ${ }^{+}$Mon $1,10^{4} / \mathrm{ml}$ & -0.101 & 0.494 & 0.114 & 0.440 & 0.065 & 0.662 \\
\hline ST2L ${ }^{+}$Mon $2,10^{4} / \mathrm{ml}$ & 0.264 & 0.070 & -0.215 & 0.142 & 0.047 & 0.750 \\
\hline ST2L ${ }^{+}$Mon $3,10^{3} / \mathrm{ml}$ & 0.203 & 0.166 & 0.310 & 0.032 & -0.133 & 0.369 \\
\hline
\end{tabular}

Abbreviations: $\mathrm{MLA}=$ minimum lumen area. Other abbreviations listed as Table I. 
Table IV. Multivariate logistic regression analysis for predictors of TCFA

\begin{tabular}{|c|c|c|c|c|}
\hline & \multicolumn{2}{|c|}{ Univariate analysis } & \multicolumn{2}{|c|}{ Multivariate analysis } \\
\hline & $\mathrm{OR}(95 \% \mathrm{CI})$ & $P$ value & $\mathrm{OR}(95 \% \mathrm{CI})$ & $P$ value \\
\hline Age $(\geq 65$ yrs $)$ & $1.07(0.30-3.78)$ & 0.92 & & \\
\hline Gender & $3.57(0.77-16.54)$ & 0.10 & & \\
\hline Smoking & $2.05(0.61-6.82)$ & 0.24 & & \\
\hline Hypertension & $0.64(0.17-2.37)$ & 0.50 & & \\
\hline Diabetes mellitus & $2.57(0.62-10.71)$ & 0.19 & & \\
\hline WBC, $10^{9} / \mathrm{L}$ & $0.97(0.70-1.34)$ & 0.85 & & \\
\hline LDL-C, mg/dl & $0.92(0.43-1.98)$ & 0.83 & & \\
\hline $\mathrm{sST} 2(>2.51 \mathrm{ng} / \mathrm{ml})$ & $0.39(0.12-1.27)$ & 0.12 & & \\
\hline Mon2(> $10.6 \%)$ & $4.20(1.23-14.37)$ & 0.02 & $1.16(0.28,4.79)$ & 0.84 \\
\hline $\mathrm{ST}^{+} / \mathrm{Mon} 2(>15.31 \%)$ & $0.07(0.02-0.29)$ & $<0.001$ & $0.07(0.02,0.29)$ & $<0.001$ \\
\hline $\mathrm{ST}^{+} / \mathrm{Mon} 2\left(>0.012 \times 10^{4} / \mathrm{ml}\right)$ & $0.37(0.11-1.25)$ & 0.11 & & \\
\hline
\end{tabular}

WBC, LDL-C, HDL-C were defined as continuous variables. P values were calculated by Wald type test.

Abbreviations: $\mathrm{WBC}=$ white blood cells, HDL-C $=$ high-density lipoprotein cholesterol, LDL-C $=$ low-density lipoprotein cholesterol, $\mathrm{OR}=$ odds ratio, other abbreviations listed as Table I. 
Supplementary Table I. Summary of fluorescently labeled antibodies

\begin{tabular}{lll}
\hline Antibody & \multicolumn{1}{c}{ Catalog } & Dillution \\
\hline APC, anti-human CD45 & Miltenyi Biotec, 130-102-544 & $1: 200$ \\
FITC, anti-human CD14 & Miltenyi Biotec, 130-110-518 & $1: 100$ \\
PE, anti-human CD16 & Miltenyi Biotec, 130-113-955 & $1: 100$ \\
PerCP-eFluor 710, anti-human & eBioscience, 46-9338-42 & $1: 50$ \\
IL-33R(ST2) & & \\
\hline
\end{tabular}


Supplementary Table II. Summary of primers for quantitative RT-PCR

\begin{tabular}{ll}
\hline Gene & Primer sequence \\
\hline ST2L (Human) & Forward: 5'-ATGGGGTTTTGGATCTTAGCAAT-3' \\
TNF- $\alpha$ (Human) & Reverse: 5'-CACGGTGTAACTAGGTTTCCTT-3' \\
IL-6 (Human) & Forward: 5'-CCT CTC TCT AAT CAG CCC TCT G-3' \\
& Reverse: 5'-GAG GAC CTG GGA GTA GAT GAG-3' \\
CD163 (Human) & Forward: 5'-ACT CAC CTC TTC AGA ACG AAT TG-3' \\
& Reverse: 5'-CCA TCT TTG GAA GGT TCA GGT TG-3' \\
Arg-1 (Human) & Forward: 5'-TTT GTC AAC TTG AGT CCC TTC AC-3' \\
& Reverse: 5'-TCC CGC TAC ACT TGT TTT CAC-3' \\
GAPDH (Human) & Forward: 5'-GTG GAA ACT TGC ATG GAC AAC-3' \\
& Reverse: 5'-AAT CCT GGC ACA TCG GGA ATC-3' \\
& Forward: 5'-GGA GCG AGA TCC CTC CAA AAT-3' \\
& Reverse: 5'-GGC TGT TGT CAT ACT TCT CAT GG-3' \\
\hline
\end{tabular}



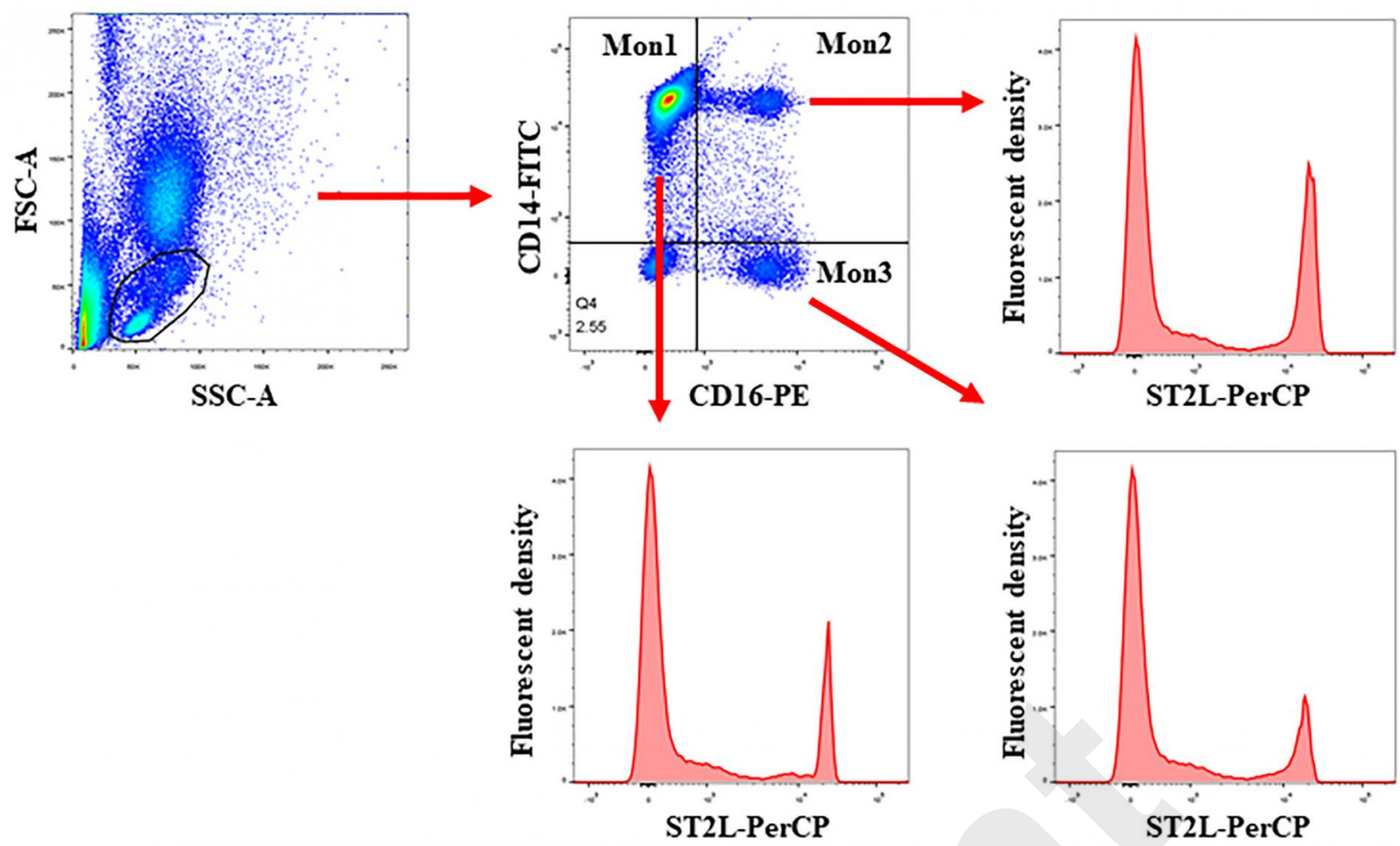

Figure 1. Gating strategy for circulating monocyte subsets and ST2L content Whole peripheral blood cells were prepared and labeled with various antibodies. CD14 was used in conjunction with CD16 to identify monocyte subsets (Mon). Mon1 was identified as CD14++CD16-, Mon2 as CD14++CD16+ and Mon3 as CD14+CD16+. ST2L antibodies were further used to stratify the percentage of ST2L positive monocyte subsets. 
A

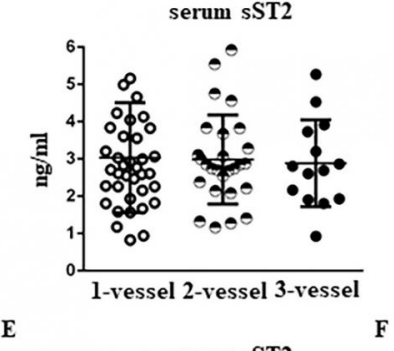

E
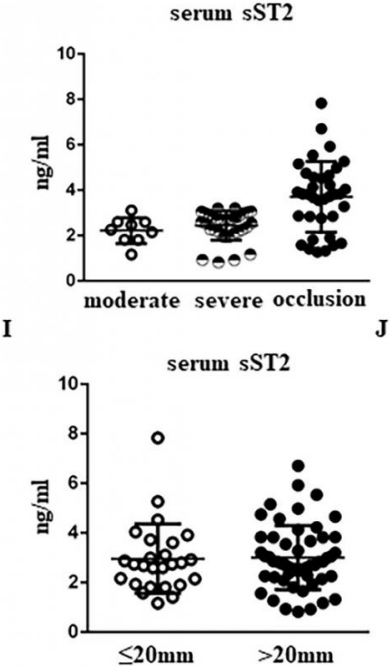

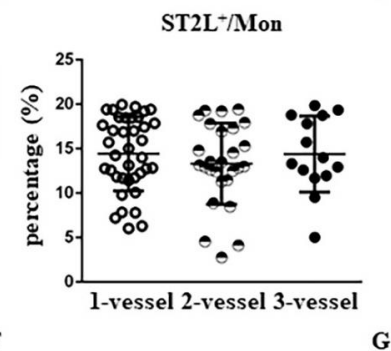

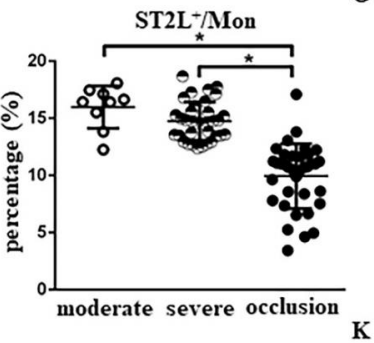

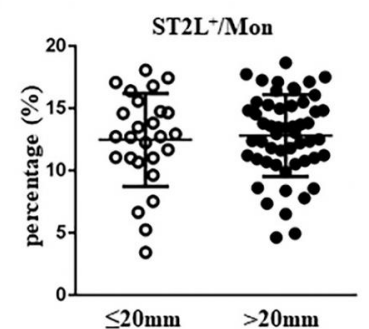

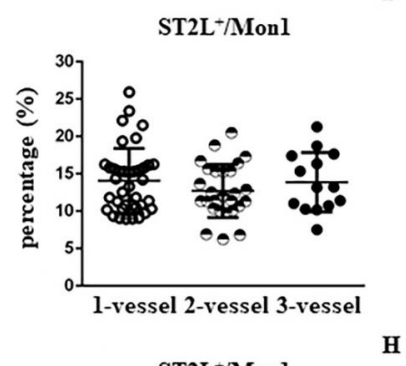
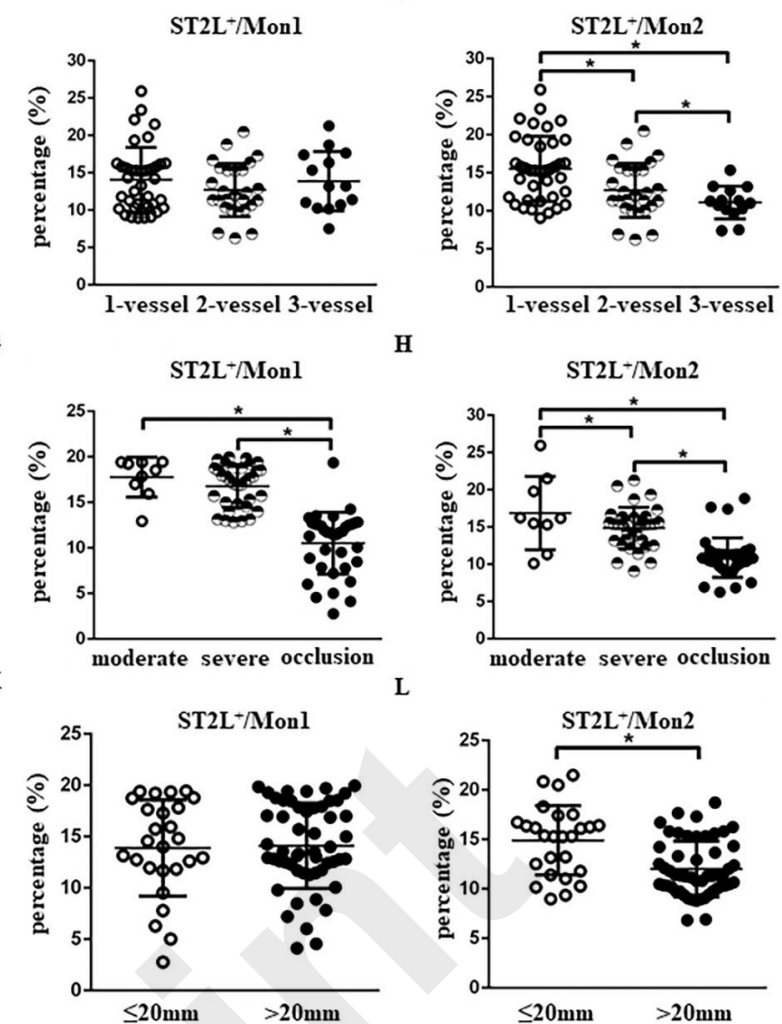

L

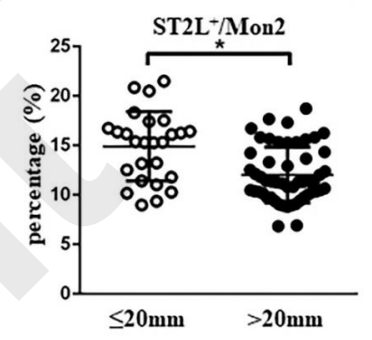

Figure 2. Comparison of serum SST2 and the percentage of ST2L expression on monocytes in patients with different severities of coronary plaques detected by angiography A-D. Comparison of serum sST2 and the percentage of ST2L expression on monocytes according to the number of stenotic vessels. E-H. Comparison of serum ST2 and the percentage of ST2L expression on monocytes according to the degree of stenosis. I-L. Comparison of serum ST2 and the percentage of ST2L expression on monocytes according to the lesion length. Abbreviations: $\mathrm{ST} 2=$ suppression of tumorigenicity $2, \mathrm{ST} 2 \mathrm{~L}=$ transmembrane ST2 isoform, Mon $=$ monocyte. ${ }^{*} \mathrm{P}<0.05$. 


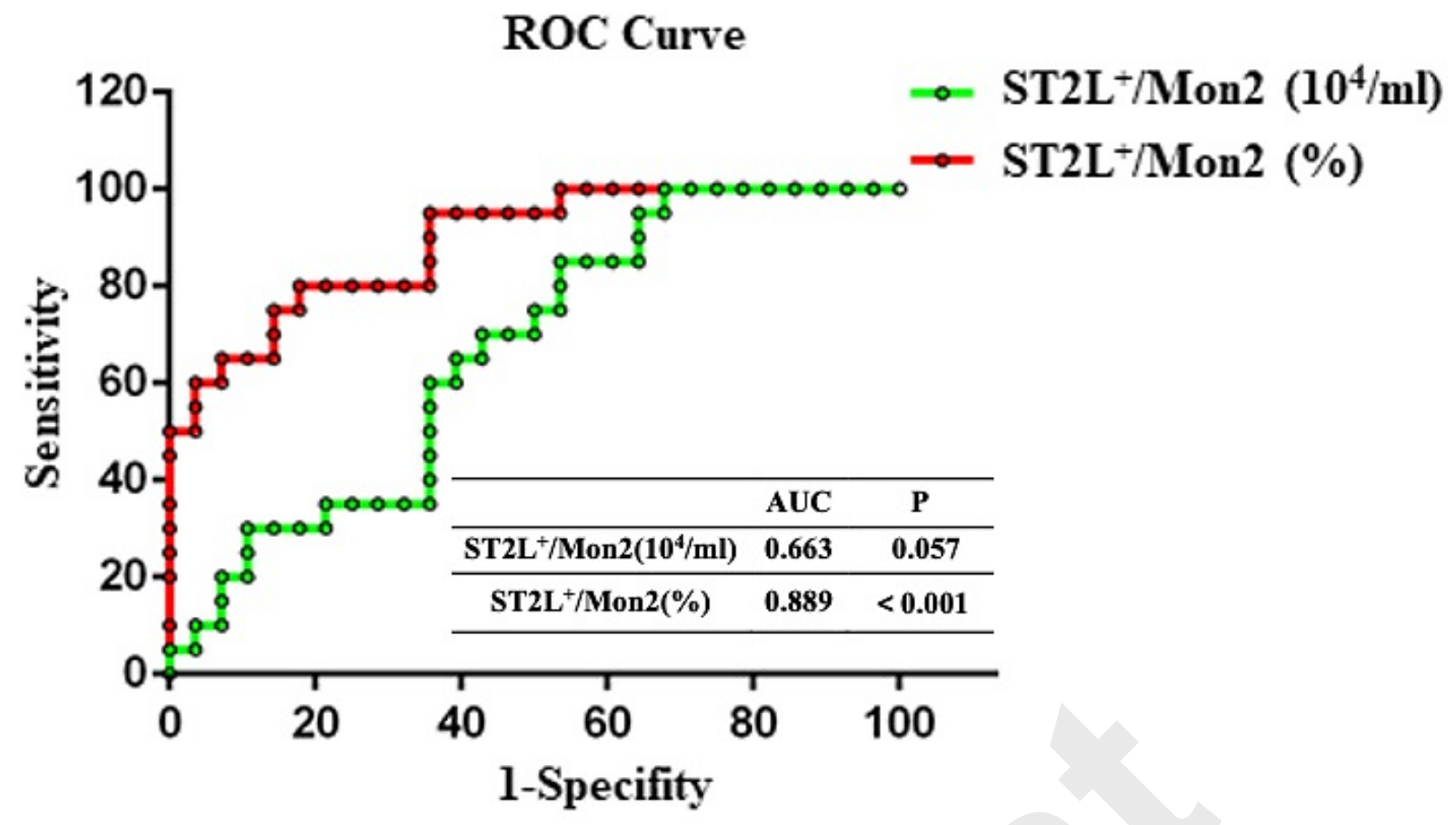

Figure 3. ROC curve depicting the distinguishing ability of absolute number and percentage of ST2L+/Mon2 for TCFA.

Abbreviations: $\mathrm{ROC}=$ receiver-operating characteristics; $\mathrm{AUC}=$ area under the curve; TCFA $=$ thin cap fibrous atheroma. 
A
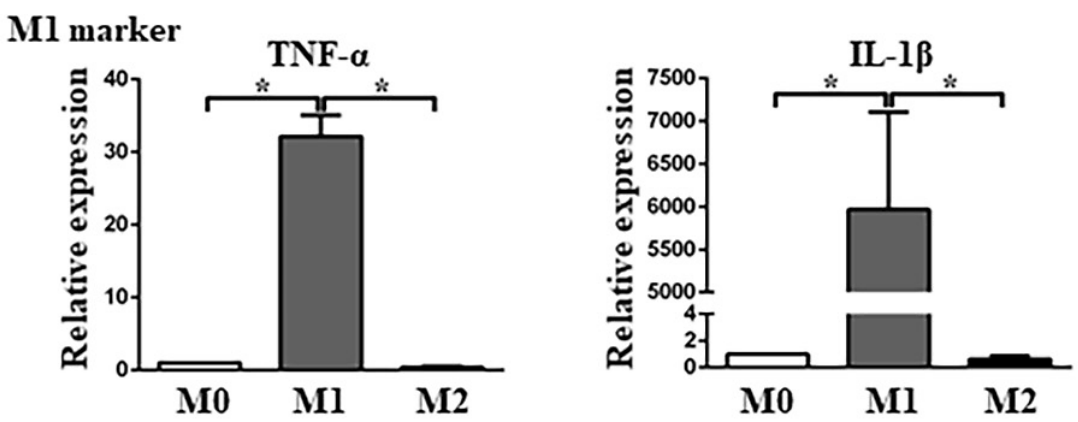

M2 marker
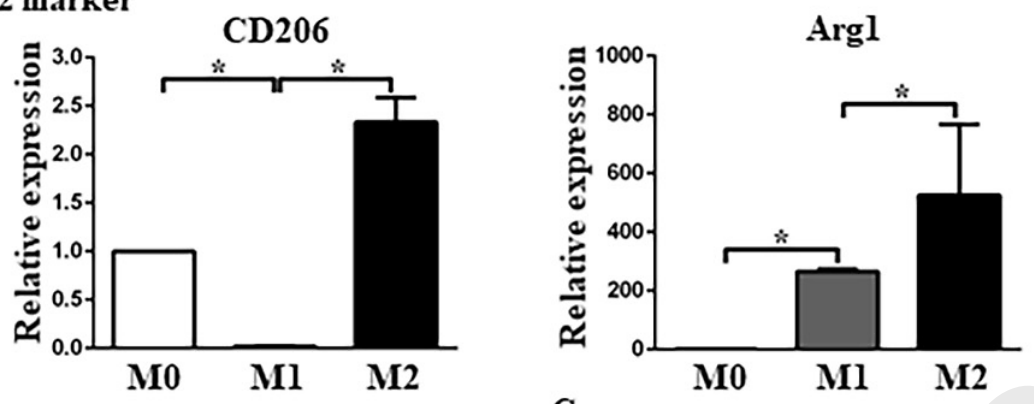

B

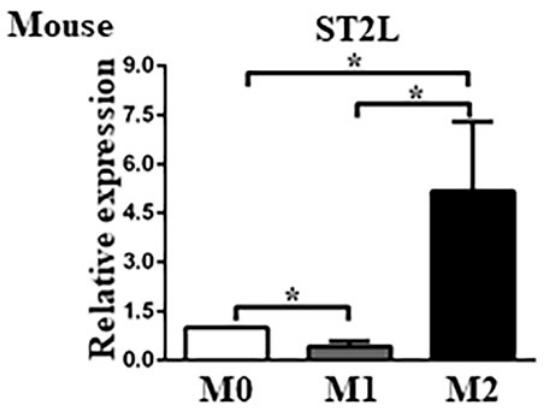

C

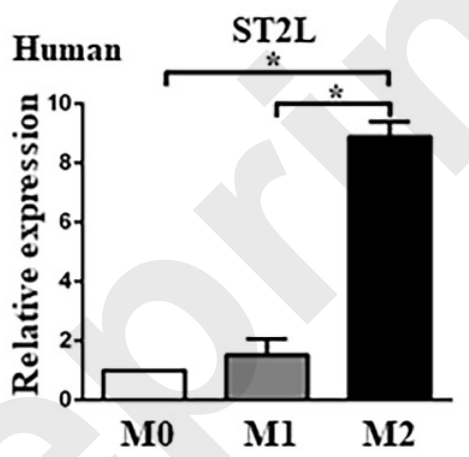

D

E
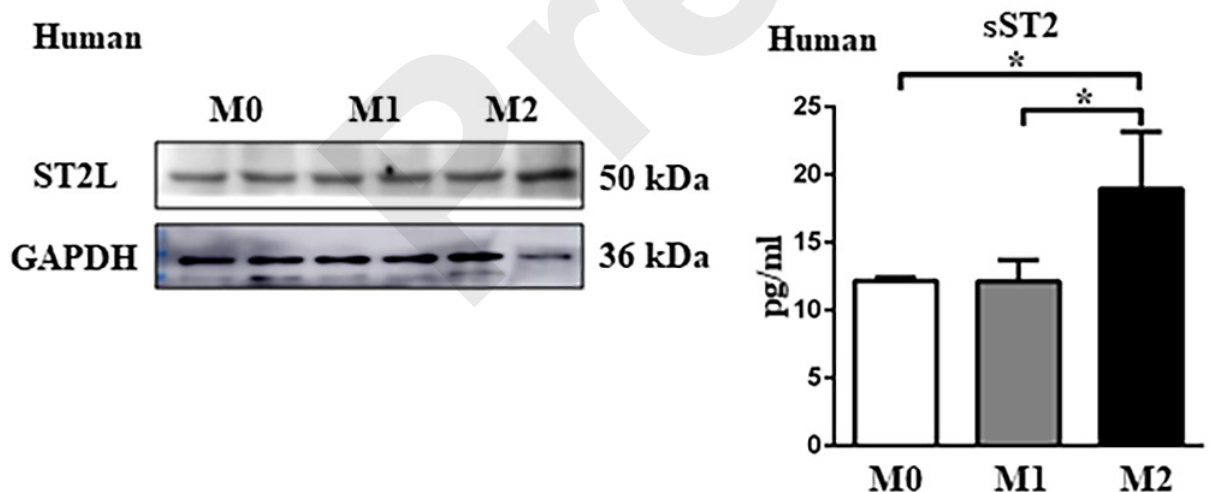

Figure 4. ST2L accumulates in M2 macrophages

A. Quantification of M1 and M2 markers in BM-derived macrophages. Macrophages were induced by LPS $(100 \mathrm{ng} / \mathrm{ml})$ and IFN-Y $(20 \mathrm{ng} / \mathrm{ml})$ for M1 polarization or by IL-4 $(20 \mathrm{ng} / \mathrm{ml})$ for M2 polarization. B and C. Quantitative PCR indicated that ST2L was upregulated in M2 polarized phenotype of mouse bone marrow-derived macrophages $(B)$ and human macrophages (C). D. Immunoblots showed the protein expression of ST2L in M0, M1 and M2 macrophages differentiated from human circulating monocytes. E. Comparison of the sST2 levels between the supernatant of M1 and M2 macrophages differentiated from human circulating monocytes. ${ }^{*} \mathrm{P}<0.05$. 